\title{
¿Cuál es la evidencia y que estrategias se recomiendan para ayudar a nuestros pacientes a dejar de fumar?
}

\section{Objetivos de la presente guía}

- Resumir los puntos relevantes de la reciente "Guía de Práctica Clínica para el tratamiento del Uso y Dependencia del Tabaco" reportado por el servicio de Salud Pública de los Estados Unidos.

- Fomentar la motivación de los médicos de atención primaria (MAP) en la intervención antitabáquica permanente como estándar de buena practica clínica.

\section{Introducción}

El tabaquismo es un grave problema de salud que ocasiona 4 millones de muertes anuales en el mundo.

Si bien en los países desarrollados el consumo de tabaco esta disminuyendo, en los países en vías de desarrollo su consumo aumenta estrepitosamente y está previsto que para el año 2025 la epidemia del tabaquismo sea responsable de 10 millones de muertes anuales, a expensas exclusivamente del aumento del consumo en los sectores mas pobres del globo. ${ }^{1}$

La Argentina no escapa a estos pronósticos y de hecho la prevalencia de tabaquismo en nuestro país se encuentra en aumento, con una prevalencia estimada del $40 \%$ en varones y de $23 \%$ en mujeres. ${ }^{2}$ Este "desplazamiento de la epidemia" desde los países desarrollados a los países en desarrollo, no ha sido fortuito ni azaroso, sino que obedece estrictamente a maniobras dirigidas para tal fin (regulaciones aduaneras, aumento de la importación, reducción de precios de los cigarrillos, maniobras de marketing y publicidad etc), con un evidente éxito en el desplazamiento del mercado del tabaco. También esta observándose un aumento del consumo en ciertas subpoblaciones como son los niños y adolescentes, las mujeres y los sectores más pobres de las sociedades, lo cual genera gran preocupación.

Si bien es cierto que las medidas necesarias para resolver este grave problema de la adicción al tabaco dependen de decisiones que exceden el marco de nuestro accionar médico, los agentes de salud y en especial los MAP debemos ser conscientes de este fenómeno epidemiológico dado que el alcance y la eficacia de nuestras intervenciones pueden ser de gran magnitud.

En 1996 se realizaron las primeras guías de manejo del Tabaquismo por la Agency for Health Research and Quality (AHRQ) que depende del US Public Health Service que analizó la evidencia bibliográfica existente hasta 1994. La aparición de nuevos y eficaces tratamientos desde entonces hasta hoy, motivaron la actualización de dichas recomendaciones originales incluyendo la evidencia publicada hasta 1999. Lo que se resume a continuación es una sintesis de las nuevas Recomendaciones, cuyo resumen fue publicado recientemente en JAMA 3, y la fuerza de las recomendaciones (A, B, C, D O E)* fue obtenida del capitulo 6:"Evidence" del reporte original de Salud Pública Americana, que diera origen al resumen publicado en JAMA, antes mencionado. ${ }^{4}$

Para la realización de estas nuevas Guías participaron 7 entidades gubernamentales y fundaciones sin fines de lucro. ${ }^{5}$

\section{¿A quienes va dirigida la guía?}

Las recomendaciones están dirigidas a tres diferentes niveles de atención y se proponen las intervenciones con documentada evidencia para cada nivel de atención.

\begin{tabular}{l|l|l}
\hline A quien va dirigido & Tipo de intervención & Lugar de implementación \\
\hline $\begin{array}{l}\text { Médicos de atención } \\
\text { primaria }\end{array}$ & Intervenciones breves & Consultorios \\
\hline $\begin{array}{l}\text { Especialistas en } \\
\text { Tabaquismo }\end{array}$ & Intervenciones Intensivas & Programas antitabáquicos \\
\hline $\begin{array}{l}\text { Administradores } \\
\text { de Salud }\end{array}$ & $\begin{array}{l}\text { Intervenciones Instit. y de } \\
\text { Sistemas de Salud }\end{array}$ & $\begin{array}{l}\text { Instituciones, prepagas } \\
\text { yotros sistemas de salud. }\end{array}$ \\
\hline
\end{tabular}

Tres grandes novedades presenta esta Guía en relación a la anterior - Todos los productos con tabaco (no solamente los cigarrillos) son igualmente devastadores para la salud de las personas.

- La mayoría de los fumadores son adictos a la nicotina y el nivel de dependencia es comparable al causado por opiáceos, anfetaminas y cocaína.

- Dada la naturaleza crónica de la adicción al tabaco y la alta frecuencia de las recaídas, el tabaquista requiere de intervenciones y cuidados médicos continuos y reiterados en el tiempo, como sucede con otras enfermedades crónicas y otras adicciones.

\section{El tabaquismo como una enfermedad crónica y tratable}

Mientras que una minoría consigue la abstinencia definitiva luego del primer intento de abandono, la mayoría de los fumadores persisten con un uso crónico y persistente de tabaco por muchos años, alternando típicamente con periodos de remisión y recaída. No entender la naturaleza del tabaquismo como una adicción crónica, puede llevar a erróneos sentimientos de frustración para el fumador y para el médico tratante, tras una recaída. Es necesario entender a esta enfermedad, como a otras enfermedades crónicas (diabetes, hipertensión, obesidad, hipercolesterolemia) en las que los pacientes requieren de cuidados médicos continuos con intervenciones reiteradas de consejo, soporte y farmacoterapia.

La alta frecuencia de las recaídas refleja la naturaleza de la dependencia y no la falla del paciente o del propio médico.

Sin embargo, a pesar de su naturaleza crónica, el tabaquismo es una enfermedad tratable. Mientras que el $7 \%$ de los fumadores consigue abandonar por sus propios medios, de un 15 a un $30 \%$ lo consigue con tratamiento. Las tasa mas altas de abandono a largo plazo se consiguen con las intervenciones intensivas y con los fármacos. No obstante las intervenciones breves realizadas por los MAP aumentan significativamente la tasa de abandono y constituyen una de las estrategias de mayor alcance.

La evidencia muestra claramente que todas las estrategias de intervención utilizadas para el cese del tabaquismo son costoefectivas dado que las personas que dejan de fumar requieren menor atención en salud y generan menores costos a los sistemas. A mayor intensidad de la intervención, mayor tasa de éxito y mayor la costoefectividad.

\section{Evaluación de los tabaquistas}

Al menos el $70 \%$ de los fumadores visitan al medico una vez al año y mas del $50 \%$ al odontólogo, por lo que dichos profesionales se encuentran en una situación de privilegio para intervenir. Un paso inicial crucial es la identificación de todos los fumadores, para luego realizar el consejo antitabáquico y una adecuada intervención.

\section{Intervenciones Breves}

Pueden ser provistas por cualquier médico pero tiene mayor impacto si son realizadas por MAP que tratan una amplia variedad de pacientes, con mayor continuidad en el tiempo.

Estas intervenciones (estimadas en 3 minutos de duración) aumentan la tasa de abandono significativamente y pueden utilizarse con toda la población: incluyendo adolescentes, embarazadas, ancianos y personas de diferentes orígenes raciales y culturales.

La realización de intervenciones breves constituye una Recomendación tipo A.*

Las intervenciones breves se deben orientar de acuerdo al momento evolutivo de la adicción al tabaco en el que se encuentra cada paciente individual.

Por ejemplo no debería colocarse una fecha para dejar de fumar y prescribirse un parche de nicotina en un paciente que no esta motivado para dejar de fumar a la brevedad. Ver figura 1. 


\section{Figura 1: Evaluación del estado tabáquico y recomendación de intervención para cada caso}

- Intervención breve para aquellos que desean dejar de fumar. Teniendo en cuenta que el $70 \%$ de los fumadores quiere dejar de fumar, esta intervención breve es de gran utilidad para los MAP. Consta de 5 pasos fundamentales que se detallan en las tablas 1 , 2 y 3.

\section{- Intervención breve para aquellos que no desean dejar de fumar.} Para ellos debería realizarse una intervención motivacional en cada consulta. La motivación es mas efectiva si se favorece la elaboración por parte del paciente, que si se abruma con información. La intervención consta de 5 pasos fundamentales que se detallan en la tabla 4.

\section{- Intervención breve para aquellos que dejaron de fumar reciente- mente.}

La intervención consiste en prevenir la recaída. Puede realizarse con una visita programada, con una llamada telefónica, o en toda circunstancia que el medico vea a su paciente. Consta de los siguientes 4 pasos, y en la tabla 5 se presentan algunos problemas frecuentes en esta etapa.

1) Reforzar la decisión de haber dejado de fumar. Feticitarlo por los logros conseguidos.

2) Revisar lo beneficios de haber abandonado. ¿Qué beneficio obtuvo hasta ahora al dejar de fumar?

3) Reforzar las razones por las que había abandonado.

4) Brindar atención de problemas residuales del abandono: manejo del estrés, exceso de peso etc.

\section{Intervenciones Intensivas}

La intensidad de la intervención guarda una relación dosis respuesta con la tasa de éxito en el abandono. Existe evidencia contundente que muestra que a mayor intensidad de las intervenciones, mayor tasa de abandono del tabaquismo y mayor costoefectividad de la intervención. Las intervenciones intensivas constituyen una Recomendación tipo " $A$ ". Las intervenciones intensivas pueden ser provistas por cualquier medico entrenado, que cuente con los recursos para ello. Así mismo las intervenciones intensivas son apropiadas para cualquier tabaquista que desee participar y no es un recurso destinado solamente a subgrupos de alto riesgo (como fumadores pesados con gran nivel de adicción) Tabla 6 .

\section{Intervenciones Institucionales}

Un porcentaje creciente de personas en muchos lugares del mundo reciben cuidados médicos a través de sistemas gerenciados de salud. En consecuencia los administradores de salud, las aseguradoras, etc, juegan roles decisivos en la administración de los recursos en salud. Estos administradores tienen un rol crucial en la promoción e implementación de sistemas y políticas institucionales que conduzcan a un tratamiento integral del tabaquismo. De hecho, estudios de investigación han comprobado que la medidas implementadas por los sistema de salud consiguen aumentar la utilización de tratamientos antitabáquicos y disminuir el numero de tabaquistas.

Sin una política a nivel institucional, los profesionales de la salud no tienen la capacidad de abordar el problema del tabaquismo de una manera consistente. Tanto como los médicos deben asumir la responsabilidad de tratar a sus pacientes tabaquistas, los administradores de salud deben asumir la responsabilidad de implementar políticas sanitarias y proveer recursos que faciliten dicho proceso. Se describen 6 estrategias de intervención institucional:

Tabla 1: Intervención breve para los pacientes que desean dejar de fumar.

\begin{tabular}{|c|c|}
\hline Propósito & Estrategia a implementar \\
\hline $\begin{array}{l}\text { 1) Conocer el estado de } \\
\text { fumador (Recom. tipo "A")* } \\
\text { e identificarlo en cada visita } \\
\text { (Recom. tipo "B" })^{*}\end{array}$ & $\begin{array}{l}\text { - Sistemáticamente poder identificar a todos los fumadores en cada visita: } \\
\text { - Registro del tabaquismo como un problema de salud: Tener un espacio consignado en la historia clínica para } \\
\text { preguntary registrar el status tabáquico actual y pasado. } \\
\text { Sistema Recordatorio: Colocar sticker identificatorio de color: " tabaquista" en la tapa de la historias de papel } \\
\text { o recordatorios electrónicos en la historias computarizadas. }\end{array}$ \\
\hline $\begin{array}{l}\text { 2) Realizar consejo } \\
\text { antitabáquico claro en cada } \\
\text { visita (Recom. tipo "A")* }\end{array}$ & $\begin{array}{l}\text { - "Creo que seria importante para usted, dejar de fumar". } \\
\text { - "Lo mas importante que puede hacer por su salud es dejar de fumar. Y yo puedo ayudarlo" } \\
\text { Ligar el tabaco con sintomas o enfermedades presentes, su impacto social y económico, el impacto en niños con } \\
\text { vivientes etc. }\end{array}$ \\
\hline $\begin{array}{l}\text { 3) Evaluar grado de } \\
\text { motivación para dejar de } \\
\text { fumar en cada visita }\end{array}$ & $\begin{array}{l}\text { - ¿Quiere dejar de fumar ahora? ¿Cree que podria hacerlo en el próximo mes? } \\
\text { - Si el paciente quiere dejar ahora: proveer un programa de abandono. } \\
\text { - Si el paciente no quiere dejar ahora: realizar una intervención motivacional. } \\
\text { Si el paciente es una embarazada, un adolescente etc. Considerar proporcionar información adicional. }\end{array}$ \\
\hline $\begin{array}{l}\text { 4) Elaborar un programa de } \\
\text { abandono del tabaquismo }\end{array}$ & $\begin{array}{l}\text { - Poner una FECHA DE ABANDONO súbita (idealmente en un lapso de } 2 \text { semanas). Constituye una Recom. tipo } \\
\text { "A"*. } \\
\text { - Notificar a familiares y amigos sobre el abandono y solicitar soporte. } \\
\text { - ESTRATEGIAS CONDUCTUALES para su manejo (ver tabla } 2 \text { para su detalle). Constituye una Recom. Tipo "B"*. } \\
\text { - Evitar el consumo de alcohol. } \\
\text { - Si hay otros convivientes fumadores, estimularlos a que abandonen o al menos evitar que fumen en su } \\
\text { presencia. } \\
\text { - OFRECER SIEMPRE FARMACOTERAPIA adecuada a cada caso, exceptuando escasas contraindicaciones. (ver } \\
\text { tabla } 3 \text { para su detalle). Constituye una Recom. tipo "A"*. } \\
\text { - Proveer material informativo suplementario. }\end{array}$ \\
\hline $\begin{array}{l}\text { 5) Programar seguimiento } \\
\text { adecuado (Recom. tipo " } C^{\prime \prime} \text { )* }\end{array}$ & $\begin{array}{l}\text { - Una primer consulta dentro la semana siguiente al día de abandono. } \\
\text { - Una segunda consulta dentro del mes siguiente al día de abandono. } \\
\text { - Propósitos de las consultas de seguimiento: Felicitar, brindar soporte, manejar los síntomas de abstinencia, } \\
\text { trabajar las situaciones de riesgo y con estrategias para manejar el stress etc. Si hubo recaída: rever las } \\
\text { circunstancias, y reprogramar una estrategia, remarcar que es muy frecuente y que sirve de aprendizaje para un futuro } \\
\text { intento. Valorar la recaida. Considerar derivación a un programa intensivo. }\end{array}$ \\
\hline
\end{tabular}


Tabla 2: Intervenciones breves para dejar de fumar: estrategias conductuales efectivas en el abandono del tabaquismo.

\begin{tabular}{|c|c|c|}
\hline $\begin{array}{l}\text { Terapias conductuales } \\
\text { Recom. Tipo " } B \text { "* }\end{array}$ & Estrategias & Ejemplos \\
\hline \multirow[t]{3}{*}{$\begin{array}{l}\text { Desarrollo de habilidadedes } \\
\text { para sostener la abstinencia } \\
\text { y entrenamiento o en la } \\
\text { resolución práctica de problemas }\end{array}$} & $\begin{array}{l}\text { Identificar SITUACIONES DE RIESGO } \\
0 \text { GATILLO de volver a fumar. }\end{array}$ & $\begin{array}{l}\text { Estar en situaciones de stress. Sentimientos negativos, estar con otros } \\
\text { fumadores, tomar alcohol, estar bajo presión o tensión, sentirse } \\
\text { angustiado o ansioso. Es fundamental que cada paciente identifique } \\
\text { las propias situaciones de riesgo. }\end{array}$ \\
\hline & $\begin{array}{l}\text { Identificar ESTRATEGIAS } \\
\text { SUSTITUTIVAS al acto de fumar. }\end{array}$ & $\begin{array}{l}\text { - Estrategias conductuales para manejar la urgencia por fumar y para } \\
\text { manejar el stress: salir a caminar, distraerse, hacer algo placentero, } \\
\text { aplicar alguna técnica de relajación, leer, etc } \\
\text { Mejorar la calidad de vida, realizar alguna actividad placentera } \\
\text { cotidianamente, realizar actividad física. }\end{array}$ \\
\hline & $\begin{array}{l}\text { Informar sobre la NATURALEZA DEL } \\
\text { TABAQUISMO y CLAVES PARA EL } \\
\text { EXITO }\end{array}$ & $\begin{array}{l}\text { - Informar sobre sintomas de abstinencia y su periodo de duración. } \\
\text { Informar sobre naturaleza adictiva de la nicotina. } \\
\text { - Informar que la urgencia por fumar cede al cabo de } 2 \text { o } 3 \text { minutos y } \\
\text { que la estrategia sustitutiva es clave para atravesar ese momento de } \\
\text { urgencia. } \\
\text { - Alertar que una sola "pitada" puede implicar una recaída. }\end{array}$ \\
\hline \multirow[t]{3}{*}{$\begin{array}{l}\text { SOPORTE DENTRO DEL PROGRAMA } \\
\text { (brindado por el MAP) }\end{array}$} & $\begin{array}{l}\text { Favorecer la ESTIMULACION PARA } \\
\text { EL ABANDONO. }\end{array}$ & $\begin{array}{l}\text { - Transmitir que existe un tratamiento efectivo para dejar de fumar. } \\
\text { - Que la mitad de la gente que alguna vez fumó, pudo dejar. } \\
\text { - Transmitir confianza en la capacidad de lograrlo. }\end{array}$ \\
\hline & $\begin{array}{l}\text { Ofrecer CUIDADO } \\
\text { Responder INQUIETUDES } \\
\text { Desmitificar TEMORES }\end{array}$ & $\begin{array}{l}\text { - Preguntar como se sienten con el hecho de dejar de fumar. } \\
\text { - Ofrecer en forma directa el deseo de ayudarlos. } \\
\text { Ser abierto a las expresiones de duda, temores o sentimientos } \\
\text { ambivalentes acerca de dejar de fumar. }\end{array}$ \\
\hline & $\begin{array}{l}\text { Favorecer la ELABORACION DEL } \\
\text { PROCESO DE ABANDONO }\end{array}$ & $\begin{array}{l}\text { Preguntar y trabajar sobre: } \\
\text { - Razones para dejar de fumar. } \\
\text { - Preocupaciones y dudas si deja de fumar. } \\
\text { - Logros conseguidos hasta ese momento. } \\
\text { - Dificultades en el proceso de abandono. }\end{array}$ \\
\hline $\begin{array}{l}\text { SOPORTE FUERA } \\
\text { DEL PROGRAMA } \\
\text { (brindado por el entorno social } \\
\text { y familiar) }\end{array}$ & $\begin{array}{l}\text { Solicitar SOPORTE SOCIALY } \\
\text { FAMILIAR }\end{array}$ & $\begin{array}{l}\text { - Trabajar sobre la utilidad de la contención familiar y social. (brindar } \\
\text { material si lo hubiera) } \\
\text { - Estimularlos a que generen un ambiente libre de tabaco en la casa y } \\
\text { en el trabajo. }\end{array}$ \\
\hline \multirow[t]{2}{*}{. } & $\begin{array}{l}\text { Facilitar el desarrollo de } \\
\text { HABILIDADES PARA CONSEGUIR } \\
\text { SOPORTE }\end{array}$ & $\begin{array}{l}\text { - Ayudar a los pacientes a identificar a las posibles personas que le } \\
\text { brinden contención y soporte en el proceso. } \\
\text { - Informarlos sobre existencia de líneas de ayudas telefónica. }\end{array}$ \\
\hline & $\begin{array}{l}\text { Estimular el SOPORTE A OTROS } \\
\text { FUMADORES }\end{array}$ & $\begin{array}{l}\text { - Ser soporte de otros fumadores para que dejen de fumar. } \\
\text { - Invitar a otras personas a cesiones para dejar de fumar. }\end{array}$ \\
\hline
\end{tabular}

1) Implementar un sistema de identificación de todos los fumadores del sistema.

2) Educar y proveer para generar profesionales interesados en intervenir en tabaquismo.

3) Destinar personal para tratar la dependencia al tabaco y realizar evaluación de su funcionamiento.

4) Promover políticas hospitalarias que fomenten y brinden servicios antitabáquicos.

5) Garantizar la cobertura del tratamiento de la dependencia al tabaco como un servicio pago para todos los afiliados, tanto el consejo y las terapias conductuales como los fármacos.

6) Remunerar económicamente a los profesionales que trabajaran en el tratamiento del tabaquismo e incluir estos jornales en los sueldos habituales.

\section{Entrenamiento a los profesionales}

Los médicos reportan que la falta de conocimiento acerca del manejo del tabaquismo es una barrera importante para intervenir. En nuestro medio, problemas frecuentes son la falta de tiempo y remuneración, una pobre formación desde la facultad y la carencia de prestigio científico que lleva implícita la dedicación a intervenciones preventivas.
Se recomienda entrenar a todo el personal de salud en las estrategias de motivación y tratamiento antitabáquico. Evidencia bibliográfica muestra que el impacto de dicho entrenamiento es mayor cuando se acompaña de otras intervenciones institucionales como por ejemplo el sistema de identificación de los pacientes tabaquistas.

El entrenamiento a los profesionales debe acompañarse de la creencia de que el tratamiento del tabaquismo constituye un estándar de calidad en la atención de la salud.

\section{Costoefectividad de las intervenciones en Tabaquismo}

Los tratamientos para dejar de fumar no son solamente efectivos desde el punto de vista clínico. También lo son desde el punto de vista económico. Desde el consejo medico, los fármacos, hasta los programas intensivos para dejar de fumar son costo-efectivos en relación a otras intervenciones medicas. Análisis de costoefectividad han mostrado que ayudar a un paciente a dejar de fumar es más costo-efectivo que tratar la hipertensión, o la hipercolesterolemia o que realizar mamografía, entre otras prácticas. Tal es así que el tratamiento para dejar de fumar ha sido llamado el "gold standard" de las intervenciones preventivas.

Cuanto más intensiva es una intervención para dejara de fumar, más 
Tabla 3: Tratamiento farmacológico.

\begin{tabular}{|c|c|c|c|c|c|c|c|}
\hline \multicolumn{2}{|l|}{$\begin{array}{l}\text { Tratamientos } \\
\text { Farmacológicos }\end{array}$} & \multirow{2}{*}{\begin{tabular}{|l}
$\begin{array}{l}\text { Contraindica- } \\
\text { ciones (CI) }\end{array}$ \\
No poseen CI \\
absolutas salvo \\
Infarto reciente \\
(2 semanas) \\
CI relativas: \\
embarazo, lactan- \\
cia y adolescentes \\
\end{tabular}} & \multirow{2}{*}{\begin{tabular}{|l|}
$\begin{array}{l}\text { Efectos } \\
\text { adversos }\end{array}$ \\
$\begin{array}{l}\text { Dispepsia, } \\
\text { síntomas } \\
\text { orales }\end{array}$ \\
\end{tabular}} & \multirow{2}{*}{$\begin{array}{l}\text { Dosis } \\
25 \text { cig. / dia: chicles } \\
(2 \mathrm{mg}) \text {. (hasta } 24 \text { chi- } \\
\text { cles/ día) } \geq 25 \text { cig./ dia: } \\
\text { chicles }(4 \mathrm{mg}) f \text { (hasta } \\
24 \text { chicles/d) }\end{array}$} & \multirow{2}{*}{\begin{tabular}{|l|}
$\begin{array}{l}\text { Duración del } \\
\text { tratamiento }\end{array}$ \\
Hasta 12 \\
semanas \\
\end{tabular}} & \multirow{2}{*}{ 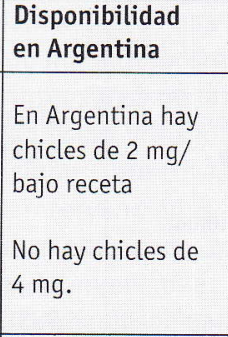 } & \multirow{2}{*}{$\begin{array}{l}\text { Costo por mes } \\
\text { s/ descuento } \\
250 \$ \text { aprox. } \\
\text { Calculando } 20 \text { chicles } \\
\text { por día. }\end{array}$} \\
\hline $\begin{array}{l}\text { Primera línea } \\
\text { Recom. tipo } \\
\text { "A" }\end{array}$ & $\begin{array}{l}\text { Chicle } \\
\text { de nicotina }\end{array}$ & & & & & & \\
\hline & \begin{tabular}{|l|} 
Parche de \\
nicotina
\end{tabular} & & $\begin{array}{l}\text { Reacción } \\
\text { local en } \\
\text { piel }\end{array}$ & $\begin{array}{ll}21 \mathrm{mg} / 24 \mathrm{hs} . & \rightarrow \\
14 / \mathrm{mg} / 24 \mathrm{hs} & \rightarrow \\
7 \mathrm{mg} / 24 \mathrm{hs} . & \rightarrow \\
15 \mathrm{mg} . / 16 \mathrm{hs} . & \rightarrow\end{array}$ & \begin{tabular}{|l|}
4 semanas \\
2 semanas \\
2 semanas \\
total: 8 \\
semanas \\
\end{tabular} & $\begin{array}{l}\text { Disponible bajo } \\
\text { receta }\end{array}$ & $\begin{array}{l}125 \$ \text { costo calculado } \\
\text { con parches de } 21 \mathrm{mg} \text {. }\end{array}$ \\
\hline & $\begin{array}{l}\text { Spray nasal } \\
\text { de nicotina }\end{array}$ & & $\begin{array}{l}\text { Irritación } \\
\text { nasal }\end{array}$ & 8-40 dosis/día & 3-6 meses & $\begin{array}{l}\text { No hay disponible } \\
\text { en Argentina }\end{array}$ & \\
\hline & \begin{tabular}{|l|} 
Inhalador \\
nicotina
\end{tabular} & & $\begin{array}{l}\text { Irritación } \\
\text { faucial }\end{array}$ & 6 a 16 puff por día & $\begin{array}{l}\text { Hasta } 6 \\
\text { meses }\end{array}$ & $\begin{array}{l}\text { No hay disponible } \\
\text { en Argentina }\end{array}$ & \\
\hline & Bupropion & $\begin{array}{l}\text { convulsiones o } \\
\text { trastornos de } \\
\text { alimentación }\end{array}$ & $\begin{array}{l}\text { Insomnio } \\
\text { boca seca }\end{array}$ & $\begin{array}{l}150 \text { los primeros } 3 \text { días, } \\
\text { luego } 300 \mathrm{mg} \text {. / día en } \\
\text { dos tomas. } \\
\text { Comenzar } 1 \text { o } 2 \text { semanas } \\
\text { antes del abandono. }\end{array}$ & $\begin{array}{l}7 \text { a } 12 \\
\text { semanas. } \\
\text { (Manteni- } \\
\text { miento hasta } \\
6 \text { meses) } f: \\
\end{array}$ & $\begin{array}{l}\text { Disponible bajo } \\
\text { receta doble }\end{array}$ & $\begin{array}{l}85 \$ \text { calculado con } 2 \\
\text { comp. de } 150 \mathrm{mg} / \text { día }\end{array}$ \\
\hline \multirow[t]{2}{*}{ Segunda linea } & $\begin{array}{l}\text { Clonidina } \\
\text { Recom. tipo } \\
\text { "A" * } \\
\end{array}$ & & $\begin{array}{l}\text { Sedación' } \\
\text { mareos, } \\
\text { boca seca }\end{array}$ & 0.15 a $0.75 \mathrm{mg} . / \mathrm{día}$ & $\begin{array}{l}3 \text { a } 10 \\
\text { semanas }\end{array}$ & $\begin{array}{l}\text { Disponible bajo } \\
\text { receta }\end{array}$ & $\begin{array}{l}45 \$ \text { calculado con } \\
0.30 \mathrm{mg} / \mathrm{dí}\end{array}$ \\
\hline & $\begin{array}{l}\text { Nortriptilina } \\
\text { Recom. tipo } \\
\text { "B"* }\end{array}$ & $\begin{array}{l}\text { Sedación boca } \\
\text { seca }\end{array}$ & & 75 a 100 mg. / día & 12 semanas & $\begin{array}{l}\text { Disponible bajo } \\
\text { receta doble } \\
\text { receta doble. }\end{array}$ & $\begin{array}{l}36 \text { \$ calculando } 80 \\
\mathrm{mg} / \text { día }\end{array}$ \\
\hline
\end{tabular}

$f$ : todos los tratamientos farmacológicos de primera línea constituyen una Recomendación tipo "A" excepto para los chicles de 4 mg que constituyen una Recomendación tipo "B", así como el mantenimiento con Bupropion.

Tabla 4: Intervención motivacional para pacientes que no desean dejar de fumar.

\begin{tabular}{l|l}
\hline Propósito & Estrategia a implementar \\
\hline $\begin{array}{l}\text { 1) Razones personales para } \\
\text { dejar de fumar }\end{array}$ & $\begin{array}{l}\text { ¿Para usted cuales podrian ser razones importantes para dejar de fumar? El impacto de la motivación será } \\
\text { mayor si se jerarquizan las propias razones del paciente. Proveer información sobre buenas razones para dejar } \\
\text { de fumar e intentar conectarlas con los síntomas que presenta el paciente. }\end{array}$ \\
\hline 2) Riesgos del tabaquismo & $\begin{array}{l}\text { ¿Cómo cree que el tabaco afecta su salud? ¿Cuáles cree que podrían ser las consecuencias de fumar? ¿Qué riesgos } \\
\text { cree puede implicarle fumar? El médico debe resaltar aquellos riesgos que son relevantes para el paciente. Informar } \\
\text { sobre el riesgo agudo y en el largo plazo. El riesgo para el medioambiente y para los convivientes. (tabaquismo pasivo, } \\
\text { aumento de la incidencia de asma y catarros en los niños). } \\
\text { Desmitificar el hecho de que fumar pocos cigarrillos o fumar en pipa es inofensivo para la salud. }\end{array}$ \\
\hline 3) Beneficios de abandonar & $\begin{array}{l}\text { ¿Cuáles cree que podrían ser los beneficios si dejara de fumar? Nuevamente, jerarquizar las prioridades del paciente. } \\
\text { Proveer información sobre los beneficios posibles: ej: mejorar la salud general, el rendimiento físico, disminuir } \\
\text { los riesgos de cáncer e infarto, tener hijos mas sanos, arrugarse mucho menos, sentir olores, ahorrar dinero etc. }\end{array}$ \\
\hline $\begin{array}{l}\text { 4) Obstáculos para } \\
\text { abandonar }\end{array}$ & $\begin{array}{l}\text { ¿Hay algo que le impide dejar de fumar? ¿Que le preocuparía si deja de fumar? ¿Le genera algún temor dejar } \\
\text { de fumar? Suelen aparecer temores al fracaso, al exceso de peso, a la pérdida del placer por fumar. } \\
\text { Ofrecer respuestas y alternativas de solución a dichos temores. }\end{array}$ \\
\hline $\begin{array}{l}\text { 5) Repetición de la } \\
\text { intervención }\end{array}$ & \begin{tabular}{l} 
pueda, el síntoma de consulta con el tabaquismo. \\
\hline
\end{tabular}
\end{tabular}

Tabla 5: Intervención breve para pacientes que dejaron de fumar recientemente.

\begin{tabular}{l|l}
\hline Problema & Respuestas a dichos problemas \\
\hline Falta de soporte & Programar visitas de seguimiento. Referir al paciente a un programa grupal. Ayudarlo a identificar soporte social en su entorno. \\
\hline Actitud negativa o depresión & Valorar necesidad de medicación antidepresiva y derivación a psicopatología. \\
\hline $\begin{array}{l}\text { Sintomas de abstinencia } \\
\text { prolongados }\end{array}$ & $\begin{array}{l}\text { Considerar la prolongación del tratamiento farmacológico o incorporar asociaciones en el caso de que no hubieran sido } \\
\text { utilizadas hasta entonces. }\end{array}$ \\
\hline Aumento de peso & $\begin{array}{l}\text { Realizar actividad física y una adecuada alimentación (desaconsejar dietas estrictas) "Normalizar" el aumento de peso } \\
\text { leve como una situación habitual en el cese tabáquico. Focalizar en el abandono tabáquico. Utilizar fármacos que retrasa } \\
\text { la aparición del exceso de peso (chicles de nicotina y bupropion). }\end{array}$ \\
\hline Pérdida de la motivación & $\begin{array}{l}\text { Repasar razones del abandono, recomendar actividades placenteras. Normalizar estos sentimientos. Prevenir que una } \\
\text { simple " pitada" puede hacer retroceder muchos logros. Repasar los beneficios conseguidos hasta el momento. }\end{array}$ \\
\hline
\end{tabular}


costoefectiva es. En otras palabras, a mayor intensidad de la intervención menor el costo por año de vida salvada. Los programas intensivos grupales mostraron el mas bajo costo por año de vida salvada que cualquier otra intervención. Pero pese a ser la mas costo- efectiva, en términos absolutos, son pocos los fumadores que acceden a estos programas (solo el $5 \%$ ) por lo que fomentar el resto de las estrategias (a través de los MAP, por ejemplo) es fundamental para aumentar el alcance de las intervenciones antitabáquicas.

Tabla 6: Intervenciones Intensivas

\begin{tabular}{|c|c|}
\hline Componentes & Estrategia para llevarlo a cabo \\
\hline Evaluación & $\begin{array}{l}\text { Debe VALORARSE LA MOTIVACION antes de ingresar a una intervención intensiva para dejar de fumar. Debe } \\
\text { recavarse información necesaria para aplicar en el tratamiento (nivel de stress, de adicción, comorbilidades, } \\
\text { intentos previos etc.). }\end{array}$ \\
\hline $\begin{array}{l}\text { Personal de salud del } \\
\text { programa }\end{array}$ & $\begin{array}{l}\text { Pueden ser PROFESIONALES DE LA SALUD DE DIFERENTES CAMPOS DE ACCION: médicos, psicólogos, } \\
\text { enfermeros etc. } \\
\text { Una estrategia podría ser: } \\
\text { UN MEDICO: aspectos vinculados a salud- enfermedad y manejo farmacológico. } \\
\text { UN PROFESIONAL NO MEDICO: terapias conductuales. }\end{array}$ \\
\hline Intensidad del programa & $\begin{array}{l}\text { Dada la fuerte relación dosis respuesta, el programa debiera consistir en AL MENOS } 4 \text { SESIONES, DE AL MENOS } \\
10 \text { MINUTOS CADA UNA, CON UN CONTACTO TOTAL DE AL MENOS } 30 \text { MINUTOS. }\end{array}$ \\
\hline Formato del programa & $\begin{array}{l}\text { Puede ser tanto GRUPAL O INDIVIDUAL. El consejo telefónico también es efectivo. El uso de material de lectura } \\
\text { es opcional. La evaluación y el seguimiento luego del abandono deben ser incluidos en el programa. }\end{array}$ \\
\hline $\begin{array}{l}\text { Tipo de consejo y terapias } \\
\text { conductuales }\end{array}$ & $\begin{array}{l}\text { ESTRATEGIAS CONDUCTUALES. Al igual que se describe en las intervenciones breves: 1) Desarrollo de habilidades } \\
\text { para el sostén de la abstinencia y entrenamiento en la resolución practica de problemas.2) Soporte dentro del } \\
\text { programa y 3) Soporte fuera del programa (ver tabla 2). }\end{array}$ \\
\hline Farmacoterapia & Ver tabla 3 \\
\hline Población a quien va dirigida & $\begin{array}{l}\text { TODOS LOS FUMADORES: siempre que fuera posible, debería utilizarse en todo fumador interesado en participar de un } \\
\text { programa intensivo y no restringirlo a los pacientes con mayor nivel de adicción o mayores intentos frustros. }\end{array}$ \\
\hline
\end{tabular}

\section{Conclusiones}

1) La dependencia al tabaco es un problema de salud crónico y a menudo requiere de intervenciones repetidas. A pesar de ello existe un tratamiento efectivo que consigue producir altas tasas de abstinencia a largo plazo.

2) Dado que existe una terapia efectiva, a todo paciente que fuma se le debe ofrecer motivación, consejo y tratamiento farmacológico para dejar de fumar.

3) Es imprescindible que la institución implemente un sistema de identificación de todos los fumadores, su documentación y su tratamiento.

4) Las intervenciones breves son efectivas, por lo tanto todo paciente fumador debe recibir al menos esta alternativa de tratamiento.

5) Existe una fuerte relación dosis respuesta entre la intensidad de las intervenciones y la tasa de éxito. Los tratamientos que implican contacto persona a persona (ya sea individual o grupal) son efectivos y su efectividad aumenta con la intensidad del tratamiento (número de sesiones, duración de las mismas, total de minutos de contacto etc.)

6) Tres tipos de intervenciones conductuales demostraron ser efectivas y deberían ser usadas como parte de los tratamientos para dejar de fumar: Desarrollo de habilidades y entrenamiento en la resolución practica de problemas, Soporte dentro del programa y Soporte fuera del programa.

7) Existen numerosas alternativas farmacológicas efectivas para el tratamiento del tabaquismo. Existen 5 fármacos de primera línea: Terapia de sustitución nicotínica (chicles, parches, spray nasal e inhalador bucal) y bupropion. Todos los pacientes, salvo escasas situaciones, deberían recibir tratamiento farmacológico.

8) El tratamiento de la dependencia al tabaco es clínicamente efectivo y costoefectivo en relación a otras practicas e intervenciones medicas. Por tal razón los sistemas de salud deberían garantizar cobertura de los tratamientos para dejar de fumar y remunerar a los profesionales que trabajan en dicha área como en cualquier otra actividad convencional.

* Ver glosario.

Dra. Verónica Schoj

Unidad de Medicina Familiar y Preventiva. Hospital Italiano de Buenos Aires.

\section{Bibliografía}

1. La Epidemia de Tabaquismo. Los gobiernos y los aspectos económicos del control del tabaco. OPS Pub. Cient. No. 577.

2. Tobacco or Health: a global status report. Country profiles by region, 1997 (http://www.cdc.gov/tobacco/who/whofirst.htm)

3. A Clinical Practice Guideline for Treating Tobacco Use and Dependence. A US Public Health Service (USPHS) Report. JAMA 2000;283;3244-3254.

4. Fiore MC, Bailey WC, Cohen SJ, et al. treating Tobacco Use and Dependence. A Clinical Practice Guideline. Rockville, Md: US Dept. of Health and Human Services, 2000. AHRQ publication No. 00-0032. Entidades Participantes: The US Agency for Healthcare Research and Quality; the Office on Smoking and Health at the Centers for Disease Control and Prevention; The National Cancer Institute; The National Institute on Drug Abuse; The National Heart, Lung, and Blood Institute, The Robert Wood Johnson Foundation; and the University of Wisconsin Medical School's Center for Tobacco Research and Intervention. 